\title{
Ensino de Estratégia na Graduação Utilizando Metodologias Ativas e Trabalhos de Campo
}

\author{
Leonardo Guimarães Garcia* \\ Departamento de Educação, Informação e Comunicação (DEDIC) da Faculdade de Filosofia, Ciências e Letras \\ de Ribeirão Preto (FFCLRP) da Universidade de São Paulo (USP-RP)
}

* Autor para correspondência: leonardogarcia@usp.br

\section{RESUMO}

O presente trabalho tem por objetivo apresentar o desenvolvimento e a aplicação de uma abordagem para o ensino de formulação de estratégias na graduação, cujos diferenciais são o uso de metodologias ativas de ensino e a realização de trabalhos de campo. Tendo como método a pesquisa-ação, este trabalho foi realizado ao longo de quatro anos e contemplou: 1) a avaliação da abordagem de ensino vigente no início do estudo; 2) seu aperfeiçoamento ao longo de quatro anos; 3) a avaliação da abordagem vigente ao final do período; e 4) a análise comparativa das avaliações inicial e final. Os resultados indicam que os aperfeiçoamentos realizados na disciplina contribuíram para a melhoria do aprendizado técnico dos alunos (isto é, seu domínio das técnicas de coleta e análise de informações estratégicas, bem como da formulação de diretrizes estratégicas para Unidades de Informação - UIs). Já o impacto da abordagem no modo como os alunos cursam a universidade diminuiu. Por outro lado, a análise sugere a consolidação de alguns pontos fortes (ligados tanto à atuação do professor quanto ao perfil da disciplina), ao mesmo tempo que indica a efetividade dos aperfeiçoamentos, uma vez que apenas uma reduzida fração das críticas e sugestões da primeira avaliação $(21,4 \%)$ se manteve na segunda.

Palavras-Chave: Ensino de Estratégia; Graduação; Biblioteconomia e Ciências da Informação e da Documentação; Metodologia Ativa; Trabalho de Campo.

\begin{abstract}
This paper aims to present the development and application of an approach to teach strategy formulation for undergraduate courses, whose differentials are the use of active learning and fieldworks. Designed based on action research, this study was conducted over four years and included: 1) the evaluation of teaching approach existing in the beginning of the study; 2) the improvements made during 4 years; 3 ) the evaluation performed at the end of the period; and 4) a comparative analysis of the results. The results indicates improvement in the students' technical learning due to the changes made in the period (namely, the students' knowledge about strategic information gathering and its analysis, and about the strategic guidelines formulation for Information Units - IUs), while the impact of approach over the way the students performing their undergraduate course declined. On the other hand, the analysis suggests the consolidation of some strengths (related either to the action of the teacher as the approach profile) at the same time that indicates the effectiveness of improvements, since only a small fraction (21.4\%) of the comments and suggestions from the first evaluation occurred at the former.
\end{abstract}

Keywords: Strategy Teaching; Undergraduate Education; Library and Information Sciences and Documentation; Active Learning; Fieldwork.

\section{Introdução}

A proposição de caminhos para o sucesso organizacional constitui-se no principal desafio da formulação de estratégias. Para tanto, diferentes vertentes teóricas têm se apresentado ao longo das últimas décadas, algumas prescritivas (baseadas em processos predeterminados para a criação da estratégia) e outras descritivas (focadas na explicação a posteriori do processo) (MINTZBERG, AHLSTRAND \& LAMPEL, 2007).

A busca do sucesso por meio da estratégia igualmente ocorre em Unidades de Informação (UIs) ${ }^{1}$ 
(BARBALHO \&BERAQUET, 1995; BARRETO et al., 1997; MACIEL \& MENDONÇA, 2006), uma vez que as mesmas também estão sujeitas a restrições e pressões similares às das demais organizações. Desse modo, visto que os profissionais da informação - egressos de cursos tais como o de Biblioteconomia e Ciência da Informação (BCI), da USP de Ribeirão Preto - são os responsáveis pela gestão das UIs, sua formação na graduação precisa prepará-los para a atividade de formulação de estratégias.

No entanto, no âmbito do BCI essa preparação torna-se particularmente difícil pois, além de não haver consenso quanto ao ensino da formulação estratégica (por existirem diferentes abordagens), nosso curso é noturno, o que estabelece restrições adicionais quanto à disponibilidade dos alunos para atividades participativas ou de campo.

Talvez em função disso, nossa revisão de literatura não pôde localizar livros, teses ou artigos que indicassem alguma alternativa prática para o ensino de formulação de estratégias, voltada especificamente para os cursos de Ciência da Informação e/ou de Biblioteconomia.

Sendo assim, o presente trabalho tem por objetivo apresentar o desenvolvimento e a aplicação de uma abordagem para o ensino da formulação de estratégias no BCI, cujos diferenciais são o uso de metodologias ativas de ensino e a realização de trabalhos de campo.

\section{O Ensino de Formulação de Estratégias na Graduação e a Metodologia Ativa}

Dada a importância da formulação de estratégias para as organizações, por um lado, e a constante evolução do seu arcabouço conceitual ao longo das últimas décadas, por outro, as universidades vêm desenvolvendo disciplinas e abordagens educacionais com o intuito de incorporarem as novidades da área e, assim, prepararem melhor seus egressos (BOWER, 2008).

Não por acaso, Fáveo et al. (2013), ao discutirem o ensino de estratégia na graduação, mencionam a grande diversidade de soluções, classificadas pelos mesmos segundo duas dimensões: a concepção/o corpo de conhecimento adotado; e as abordagens/ técnicas de ensino usadas. Essa mesma diversidade é encontrada no ensino de estratégia na pósgraduação (VILLAR \& WALTER, 2014).

Nesse âmbito, a literatura discute formas de integrar teoria e prática com o intuito de ampliar o aprendizado dos alunos (JENNINGS, 1996; GREINER, BHAMBRI \& CUMMINGS, 2003; HARO \& TURGUT, 2012). Essa perspectiva é convergente com as metodologias ativas, cuja essência se baseia no envolvimento dos alunos na resolução de um problema prático, com autonomia e responsabilização por sua própria aprendizagem (RICHARTZ, 2015).

Aplicadas em diversas áreas, incluindo a de informação (SODERDAHL, 2011), as metodologias ativas têm se mostrado eficazes na ampliação da qualidade do aprendizado (LINTON et al., 2014). Suas diversas modalidades expressam flexibilidade e adaptabilidade a diferentes contextos e disciplinas de graduação, favorecendo a inovação e o surgimento de concepções de ensino mais centradas nos alunos (RUIZ-PRIMO et al., 2011).

\section{Método}

Tendo em vista o objetivo de desenvolver e aplicar uma solução para o problema real do ensino da formulação de estratégias no BCI, este trabalho exigiu a participação não só do pesquisador, mas também de seus alunos. Sendo assim, o método de pesquisa adotado foi a pesquisa-ação que, segundo Thiollent (2009, p. 16), é voltado a investigações:

[...] com base empírica que é concebida e realizada em estreita associação com uma ação ou com a resolução de um problema coletivo e no qual os pesquisadores e os participantes representativos da situação ou problema estão envolvidos de modo cooperativo ou participativo.

O delineamento da pesquisa seguiu as etapas do quadro 1. 


\begin{tabular}{|c|c|}
\hline $\begin{array}{c}\text { Fases da } \\
\text { Pesquisa-Ação }\end{array}$ & Objetivo Geral da Fase \\
\hline Fase exploratória & $\begin{array}{c}\text { Delimitar o campo de } \\
\text { investigação e estabelecer } \\
\text { as expectativas dos } \\
\text { participantes do processo } \\
\text { investigado }\end{array}$ \\
\hline
\end{tabular}

Formulação

do Problema e

Desenvolvimento

do Trabalho

Propor o problema de pesquisa com clareza e precisão, bem como o modo com que foi desenvolvido

Determinar a amostragem adequada à coleta de dados

Seleção de Amostra

Aplicar à amostra selecionada os instrumentos de coleta de dados

Análise dos Dados

Analisar os dados coletados

Apresentar a pesquisa e seu

Realização de

Seminários

Divulgação dos

Resultados melhorias

Publicar os resultados junto à comunidade científica da área
Levantamento bibliográfico relativo ao ensino de formulação de estratégias na graduação; levantamento informal das percepções dos alunos (em 2010), quanto ao projeto original da disciplina Planejamento e Informação do BCI, responsável pelo ensino de formulação de estratégias no âmbito do curso.

Da fase exploratória surgiu o objetivo da pesquisa, que foi o de desenvolver e aplicar uma nova abordagem para o ensino da formulação de estratégias no $\mathrm{BCI}$, baseada em metodologias ativas. Disso resultou, em 2011, uma nova proposta para a disciplina Planejamento e Informação, a qual foi implementada e avaliada pelos alunos naquele ano. Desde então, aperfeiçoou-se a disciplina, até receber nova avaliação em 2015, pelo mesmo instrumento de 2011, para se observar a evolução ocorrida no período.

Todos os alunos de 2011 e 2015 foram convidados a participar, voluntária e anonimamente, da pesquisa.

Ao final dos semestres letivos de 2011 e 2015, o instrumento de coleta foi disponibilizado via internet (questionário on-line) aos alunos para que pudessem avaliar a disciplina. O próprio aplicativo do instrumento armazenou os dados numa planilha, da qual foram extraídos os dados para a análise.

Os dados foram analisados com o intuito de se observar o processo evolutivo da disciplina no período (os resultados dessa análise estão descritos na seção 4 deste artigo).

Apresentação da proposta do estudo e de seus resultados preliminares no I Congresso de Graduação da USP, realizado em 2015.

Escrita do presente trabalho.

Quadro 1 - Fases da pesquisa-ação aplicadas ao presente trabalho. Fonte: Elaborado pelo autor.

\section{Resultados e Discussão}

Descrição da Abordagem de Ensino Vigente no Momento da Primeira Avaliação

No BCI, o ensino de formulação de estratégias está a cargo da disciplina Planejamento e Informação. Desde 2011 (ano de início do uso da nova abordagem), ela vem sendo ministrada com o auxílio de uma das bibliotecas do campus de Ribeirão Preto.

A biblioteca funciona como o "laboratório" da disciplina, por meio do qual os alunos realizam trabalhos de campo, direcionados a atividades 
essenciais para a formulação estratégica (coleta de dados em fontes formais, entrevistas e observação in loco). As informações coletadas na biblioteca são tratadas, convertidas em forças, fraquezas, ameaças e oportunidades e, por fim, submetidas à análise $\mathrm{SWOT}^{2}$ para a criação da estratégia.

Em 2011, os componentes do plano da disciplina eram os seguintes:

- Objetivo: Apresentar aos alunos os conceitos e as técnicas fundamentais do processo de planejamento estratégico de Unidades de Informação (UIs).

- Metodologia de Ensino: Aulas expositivas, com momentos de discussão coletiva dos conceitos abordados. Realização de um projeto prático, em grupo, e de trabalhos de campo com o intuito de se formular a estratégia de uma UI real.

- Avaliação do Processo de Aprendizagem: A nota na disciplina será formada pela média das notas do relatório parcial (20\% do total) e final do projeto (30\% do total), e da prova escrita $(50 \%$ do total).

- Gonteúdo Programático: Planejamento e as Unidades de Informação (UIs); do Planejamento ao Planejamento Estratégico $(\mathrm{PE}) \mathrm{de}$ UIs; Diagnóstico interno com foco no PE de UIs; Diagnóstico externo com foco no PE de UIs; Técnica SWOT: visão geral da técnica; a descoberta e a análise das forças, fraquezas, ameaças e oportunidades da UI; formulação e escolha da estratégia mais adequada à UI; criação do plano estratégico para a UI.

- Referências: vide quadro 2.

Após ministrar a disciplina com essa abordagem, decidiu-se promover uma avaliação da mesma pelos alunos utilizando um questionário on-line. De forma anônima e espontânea, 21 dos 41 alunos da disciplina $(51,2 \%)$ responderam seis perguntas: cinco mostradas no Gráfico 1 e uma última, para críticas, sugestões e elogios, cujas respostas são discutidas posteriormente.

Da figura apreende-se que a opinião dominante foi a de que a disciplina alcançou resultados satisfatórios (em média, o percentual do estrato "satisfatoriamente" foi de 53,7\%, contra 39,5\% do estrato "muito"). Apesar de positivos, esses resultados evidenciavam a necessidade de melhorias, sobretudo quanto aos conteúdos técnicos da disciplina.

\section{O Processo de Aperfeiçoamento da Disciplina ao Longo dos Quatro anos (de 2011 a 2015)}

A partir de 2011, uma série de aperfeiçoamentos foram introduzidos na disciplina, ocasionando o amadurecimento da sua concepção, conteúdos e ferramentas. Esse processo evolutivo continuou nos anos seguintes, de modo que, em 2015, decidimos realizar um balanço

ALMEIDA, M. C. B. de. Planejamento de Bibliotecas e Serviços de Informação. Brasília: Briquet de Lemos Livros, 2000.

BARBAlHo, C. R. S. \& BERAQUET, V. S. M. Planejamento Estratégico para Unidades de Informação. São Paulo: Polis/APB, 1995.

FUNDAÇÃO NACIONAL DA QUALIDADE - FNQ. Cadernos Compromisso com a Excelência: Estratégias e Planos. Disponível em: <http://www.fnq.org.br/site/694/default.aspx>. Acessado em 12 jul. 2010.

BARRETO, A. R. et al. Gestão de Unidades de Informação. Curitiba: Tecpar; Brasília: Ibict, 1997.

FNQ. Critérios Compromisso com a Excelência e Rumo à Excelência. Disponível em: $<\mathrm{http} / / /$ www.fnq.org.br/Portals/_FNQ/Documents/RumoExcelencia2008_ebook.pdf>. Acessado em 12 jul. 2010

TARAPANOFF, K. (org.). Inteligência Organizacional e Competitiva. Brasília: Editora UnB, 2001.

MINTZBERG, H.; AHLSTRAND, B. \& LAMPEL, J. Safäri de Estratégia: um Roteiro pela Selva do Planejamento Estratégico. Porto Alegre: Bookman, 2000.

FNQ. Guia de Autoavaliação. Disponivel em:

$<$ http://www.fnq.org.br/pdf/CadernosExcelencia2008_09_avaliacao.pdf $>$. Acessado em 8 fev. 2011.

Quadro 2 - Referências utilizadas na disciplina Planejamento e Informação, em 2011. Fonte: Elaborado pelo autor. 


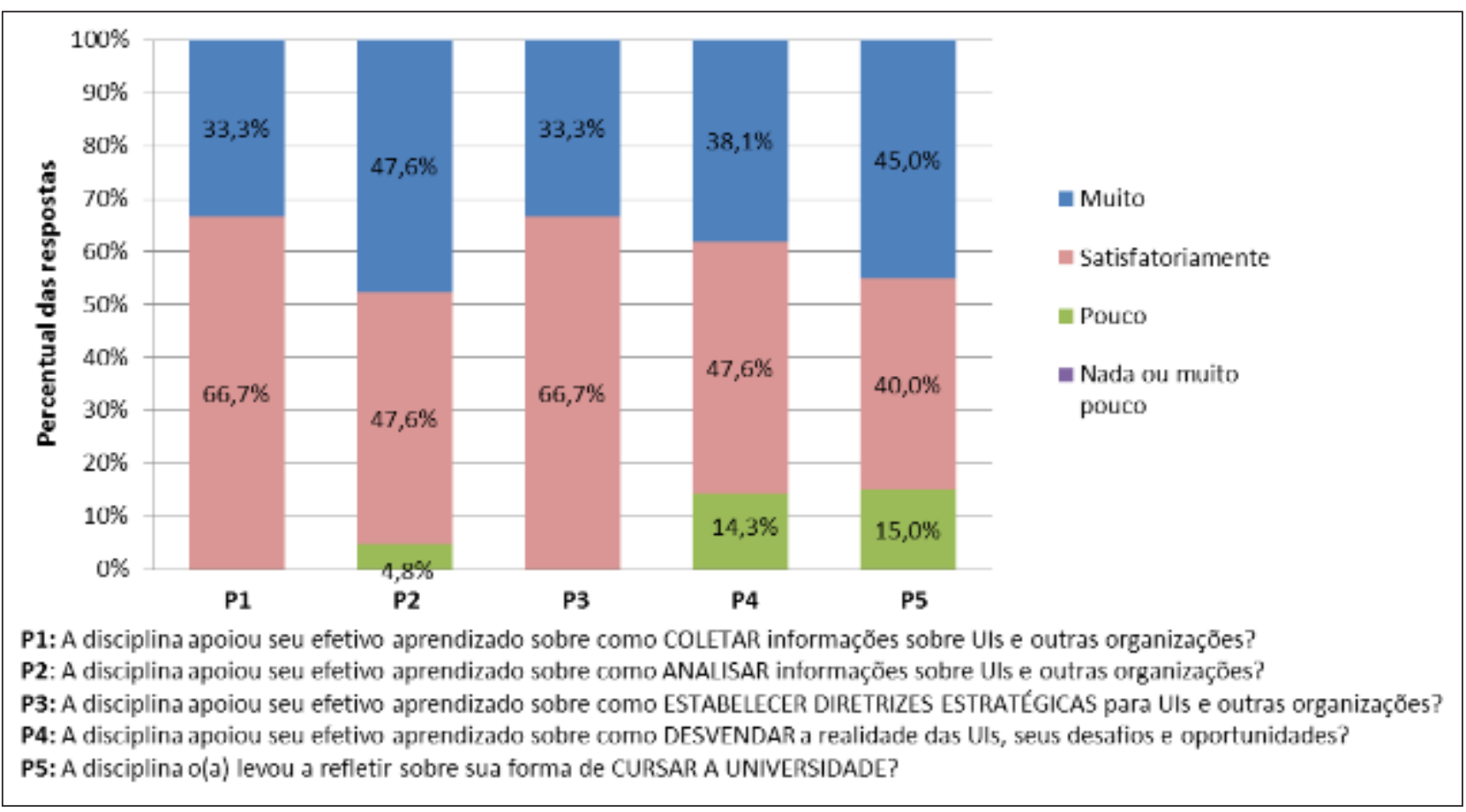

Gráfico 1 - Respostas da primeira avaliação da disciplina Planejamento e Informação. Fonte: Elaborado pelo autor.

dessas mudanças. Inicialmente, fez-se uma análise comparativa entre as versões dos planos e dos conteúdos ministrados em 2011 e 2015. O resultado dessa análise indicou três eixos principais de mudança:

- Metodologia de Ensino: Com a duplicação do número de créditos da disciplina em 2012 (que passou de 2 para 4), houve um grande aumento no número de atividades práticas realizadas em sala de aula, gerando impacto positivo sobre o aprendizado dos conteúdos técnicos. Ao longo dos anos, houve uma crescente sistematização do processo de projeto, com a criação de roteiros, modelos de relatórios e exemplos dos resultados esperados para as atividades. Houve ainda uma mudança gradual na forma com que o projeto era encarado: ainda que tratasse da formulação de estratégias para UIs, foram sendo introduzidas considerações e discussões com o intuito de ampliar o escopo de aplicação para outros tipos de organização.

- Avaliação do Processo de Aprendizagem: A nota final de cada aluno passou a ser formada exclusivamente por suas notas nas atividades ao longo do projeto. Mesmo o aluno atuando em grupo, as notas das atividades passaram a ser individuais. Para que cada um pudesse relatar suas ações, foi criado o diário de bordo (diário semanal das atividades, preenchido individualmente e validado pelo relator do grupo).

- Conteúdo Programático: O conteúdo da disciplina foi sistematicamente lapidado, e um terço da bibliografia foi alterado ao longo do período do estudo. O principal conteúdo introduzido relacionava-se à identificação do que é sucesso para uma UI. Disso, resultou o surgimento de uma nova rodada de entrevistas com todos os membros da biblioteca, para se descobrir de qual noção de sucesso todos compartilhavam.

\section{Comparando os Resultados das Avaliações de 2011 e 2015}

De modo a tornar a comparação mais consistente, decidiu-se repetir a avaliação da disciplina nos moldes daquela realizada em 2011. Em 2015 havia dezenove alunos matriculados, dos quais catorze $(73,4 \%)$ responderam, de forma voluntária e espontânea, o mesmo questionário de 2011. Os novos resultados, comparados aos da primeira avaliação, aparecem no Gráfico 2. 


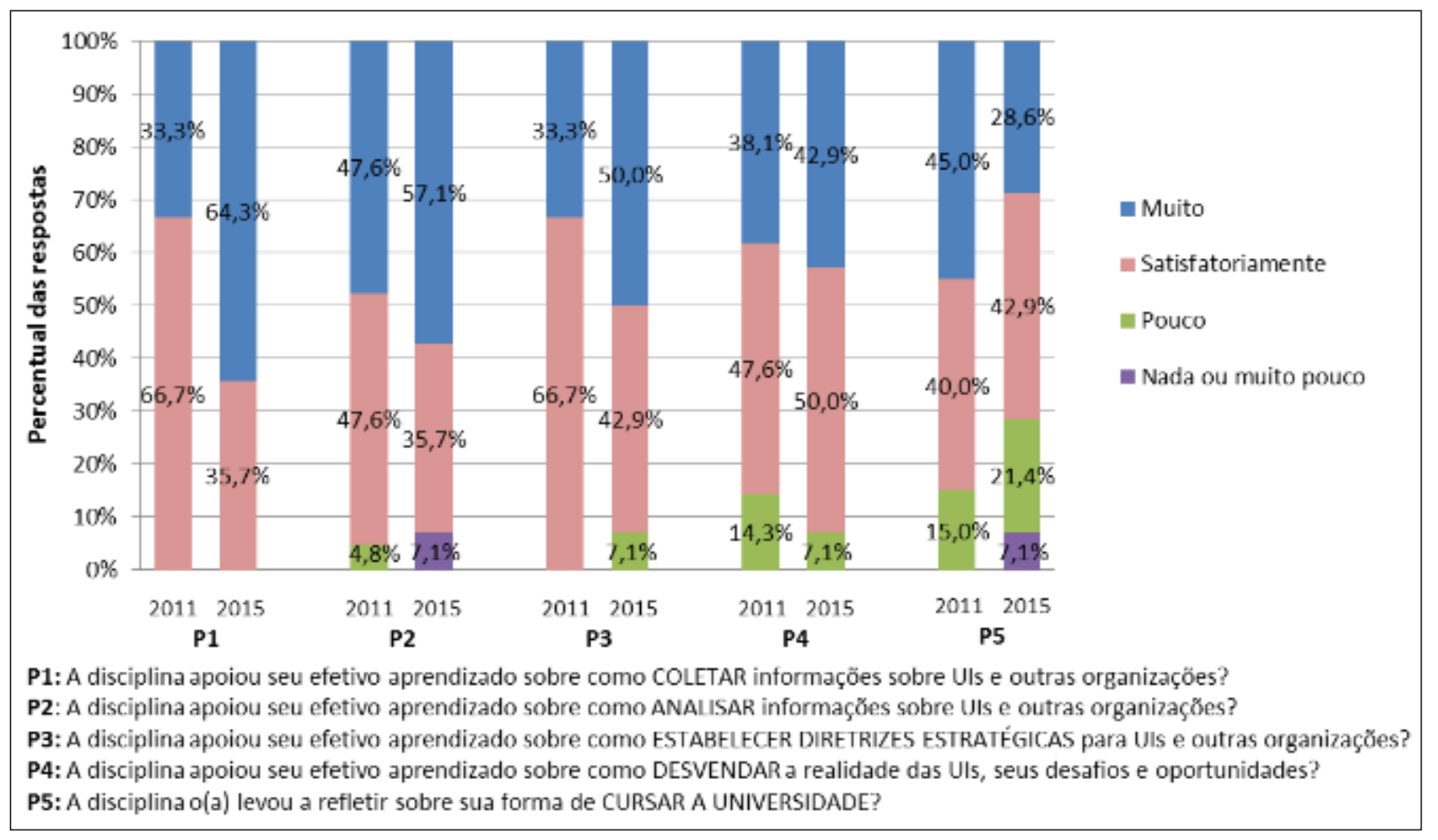

Gráfico 2 - Comparação das avaliações da disciplina em 2011 e 2015. Fonte: Elaborado pelo autor.

Os resultados indicam que os aperfeiçoamentos realizados na disciplina contribuíram para a melhoria do aprendizado técnico dos alunos (perguntas P1, P2, P3 e P4), uma vez que o percentual do estrato "muito" aumentou para as quatro perguntas $^{3}$. Já o impacto da disciplina no modo como os alunos cursam a universidade (pergunta P5) diminuiu.

Os aperfeiçoamentos também tiveram efeito positivo sobre as críticas, sugestões e elogios expressos em 2011, conforme mostram os Quadros 3, 4 e 5.

Das cinco críticas formuladas em 2011 (reduzido número de créditos da disciplina; foco exclusivo em bibliotecas universitárias; falhas nos roteiros de atividades; conteúdo teórico reduzido e um pouco repetitivo; e alta carga de trabalho), apenas a última reapareceu em 2015. Além disso, somente duas novas críticas, ambas com baixa ocorrência, surgiram em 2015. Esse resultado sugere a eliminação dos problemas em função do aperfeiçoamento da disciplina. Por outro lado, observa-se uma concentração das críticas em torno de um único ponto (a alta carga de trabalho). Essa crítica já era a mais importante de 2011, e manteve esse posto em 2015, o que indica a necessidade de uma nova reflexão sobre essa questão.

Assim como para as críticas, o quadro 4 indica a diminuição da diversidade de sugestões (das nove apresentadas em $2011^{4}$, apenas duas reapareceram, e somente duas novas - ambas com baixa ocorrência - surgiram). Acreditamos que esse resultado indica que as intervenções ao longo dos quatro anos tenham resolvido problemas diagnosticados em 2011.

Diferente das críticas e sugestões, é desejável o aumento do número de elogios reincidentes, uma vez que indicaria a consolidação de pontos positivos da abordagem de ensino. Sendo assim, o quadro 5 mostra quatro pontos de destaque da abordagem (El1, El2, El3 e El4), ligados tanto à atuação do professor quanto ao perfil da abordagem adotada.

\section{Considerações Finais}

Este artigo apresenta a descrição e o aperfeiçoamento de uma abordagem para o ensino 


\begin{tabular}{|r|c|c|c|}
\hline$\#$ & Críticas ocorridas em 2011 e reincidentes em 2015 & Percentual & Viés \\
\hline Cr1 & Alta carga de trabalho & $71,4 \%$ & Negativo \\
\hline$\#$ & Críticas ocorridas apenas em 2015 & Percentual & Viés \\
\hline Cr2 & Dificuldades em reunir os grupos fora da sala de aula & $14,3 \%$ & \\
\hline Cr3 & Dificuldade de acesso às informações necessárias ao projeto & $14,3 \%$ & \\
\hline & TOTAL & $100 \%$ & \\
\hline
\end{tabular}

Quadro 3 - Críticas feitas pelos alunos à disciplina Planejamento e Informação. Fonte: Elaborado pelo autor.

\begin{tabular}{|c|c|c|c|}
\hline$\#$ & Sugestões ocorridas em 2011 e reincidentes em 2015 & Ocorrência & Viés \\
\hline Sg1 & Aumentar o número de créditos da disciplina & $25,0 \%$ & Positivo \\
\hline Sg2 & Abranger outras UIs e outros tipos de organização & $25,0 \%$ & Negativo \\
\hline$\#$ & Sugestões ocorridas apenas em 2015 & Ocorrência & Viés \\
\hline Sg3 & Aumentar o tempo dedicado à aplicação da técnica SWOT & $25,0 \%$ & \\
\hline Sg4 & Obter sala com mobiliário adequado ao trabalho em grupo & $25,0 \%$ & \\
\hline & TOTAL & $100 \%$ & \\
\hline
\end{tabular}

Quadro 4 - Sugestões feitas pelos alunos à disciplina Planejamento e Informação. Fonte: Elaborado pelo autor.

\begin{tabular}{|c|c|c|c|}
\hline \# & Elogios ocorridos em 2011 e reincidentes em 2015 & Ocorrência & Viés \\
\hline El1 & Boa didática & $14,3 \%$ & \\
\hline $\mathrm{El} 2$ & Dedicação, comprometimento e competência do professor & $7,1 \%$ & \\
\hline $\mathrm{El} 3$ & Contribuição da disciplina em sua formação técnica & $7,1 \%$ & \\
\hline El4 & $\begin{array}{l}\text { Oportunidade de revisão e retomada de conteúdos de } \\
\text { outras disciplinas }\end{array}$ & $7,1 \%$ & \\
\hline \# & Elogios ocorridos apenas em 2015 & Ocorrência & Viés \\
\hline El5 & $\begin{array}{l}\text { Contribuição da disciplina no desenvolvimento pessoal dos } \\
\text { alunos }\end{array}$ & $35,7 \%$ & \\
\hline El6 & $\begin{array}{l}\text { Contribuição da disciplina no modo como os alunos cursam } \\
\text { a universidade }\end{array}$ & $21,4 \%$ & \\
\hline \multirow[t]{2}{*}{ El7 } & Aumento do interesse pela área administrativa de UIs & $7,1 \%$ & \\
\hline & TOTAL & $100 \%$ & \\
\hline
\end{tabular}

Quadro 5 - Elogios feitos pelos alunos à disciplina Planejamento e Informação. Fonte: Elaborado pelo autor. 
de formulação de estratégias a alunos do BCI, baseada em metodologias ativas de ensino e em trabalhos de campo. Para tanto, foram discutidos os componentes da disciplina Planejamento e Informação, responsável pelo ensino desse conteúdo, bem como os resultados de avaliações da mesma, realizadas em 2011 e 2015.

Os resultados indicam que os aperfeiçoamentos efetuados na disciplina contribuíram para a melhoria do aprendizado técnico dos alunos. Já o impacto positivo da abordagem no modo como os alunos cursam a universidade diminuiu. Por outro lado, a análise sugere a consolidação de alguns pontos fortes da abordagem (ligados tanto à atuação do professor quanto ao perfil da abordagem adotada), ao mesmo tempo que aponta a efetividade dos aperfeiçoamentos, visto que apenas uma reduzida fração das críticas e sugestões ocorridas na primeira avaliação $(21,4 \%)$ se repetiu na segunda, e as novas críticas e sugestões foram em pequeno número e baixo percentual.

O conjunto dos resultados, aliado à descrição, ainda que breve, da abordagem de ensino da formulação de estratégias no BCI, nos leva a crer na possibilidade de aplicação dessas concepções e ideias a outros cursos de Biblioteconomia e Giência da Informação, uma vez que as publicações sobre o tema voltadas exclusivamente a essa área são exíguas. Mais do que isso: acreditamos que a abordagem aqui apresentada tem potencial de extrapolação para outros cursos de graduação, haja vista o interesse da área de ensino de formulação estratégica no uso de metodologias ativas.

Em suma, o presente estudo reitera a ideia de que a inserção das metodologias ativas na graduação qualifica a reformulação curricular e moderniza o ensino sem abrir mão da efetividade. Sabemos que as disciplinas servem à formação de jovens cujo perfil se transforma continuamente. Sendo nosso papel enquanto educadores o de promover o melhor contexto de aprendizagem possível, a atualização de nossas disciplinas pelo emprego das metodologias ativas apresenta-se como excelente oportunidade.

\section{Notas}

1 Organizações vinculadas a uma entidade mantenedora (ou, em alguns casos, independentes e autônomas), cujo propósito é a obtenção, o tratamento, a organização e a disseminação de informações. São exemplos de unidades de informação as bibliotecas, os centros de documentação, os núcleos de informação empresarial, dentre outros.

2 Ferramenta para a formulação de estratégias "capaz de abordar tanto o ambiente externo como o ambiente interno da organização em termos de oportunidades e ameaças exógenas e de forças e fragilidades endógenas" (CHIAVENATO \& SAPIRO, 2009, p. 181).

3 A mesma conclusão torna-se menos evidente quando analisamos a soma dos estratos "nada ou muito pouco" e "pouco" em 2011 e 2015. Em P1, esses estratos não ocorrem em 2011 ou 2015. Em P2, vemos um ínfimo aumento da soma no período. Em P3, a soma aumenta e em $\mathrm{P} 4$, diminui. Uma vez que os percentuais de variação dessa soma de estratos inferiores são bem menores que a variação positiva do estrato "muito", consideramos válida a conclusão de que os aperfeiçoamentos realizados na disciplina contribuíram para a melhoria do aprendizado técnico dos alunos.

4 Aumentar o número de créditos da disciplina; abranger outras UIs e outros tipos de organização; ampliar o tempo de execução das atividades do projeto em sala de aula; disciplinar e facilitar o acesso à UI; melhorar o preparo dos alunos para a coleta de informações; incrementar a organização dos documentos do projeto no repositório; apoiar mais o trabalho de finalização da estratégia dos grupos; firmar convênio com a UI analisada; e tornar a disciplina optativa.

\section{Referências Bibliográficas}

BARBALHO, C. R. S. \& BERAQUET, V. S. M. Planejamento Estratégico para Unidades de Informação. São Paulo: Polis/APB, 1995.

BARRETO, A. R.; SANTOS JUNIOR, J.; CARVALHO, E. R. de; RAMOS, P. A. B. Gestão de 
Unidades de Informação: Manual. Curitiba: Tecpar; Brasília: Ibict, 1997.

BOWER, J. L. "The Teaching of Strategy: from General Manager to Analyst and Back Again?". Journal of Management Inquiry, vol. 17, n. 4, dez. 2008, pp. 269-275.

CHIAVENATO, I. \& SAPIRO, A. Planejamento Estratégico: Fundamentos e Aplicações. 2 ed. Rio de Janeiro: Elsevier, 2009.

FÁVEO, J. D.; VILLAR, E. G. \& WALTER, S. A. "Desenvolvimento do Ensino de Estratégia em Cursos de Graduação em Administração de Instituições de Ensino Superior pela Perspectiva de Professores e Alunos". Seminários em Administração, 16, 2013, São Paulo. Anais... São Paulo: FEA-USP, 2013, pp. 1-15.

GREINER,L.E.;BHAMBRI,A.\&CUMMINGS, T. G. "Searching for a Strategy to Teach Strategy". Academy of Management Learning and Education, vol. 2, n. 4, dez. 2003, pp. 402-420.

HARO, S. P. \& TURGUT, G. "Expanded Strategy Simulations: Developing Better Managers". Journal of Management Development, vol. 31, n. 3, 2012, pp. 209-220.

JENNINGS, D. "Strategic Management and the Case Method". Journal of Management Development, vol. 15, n. 9, 1996, pp. 4-12.

LINTON, D. L.; PANGLE, W. M.; WYATT, K. H.; POWELL, K. N.; SHERWOOD, R. E. "Identifying Key Features of Effective Active Learning: the Effects of Writing and Peer Discussion". Life Sciences Education, vol. 13, n. 3, set. 2014, pp. 469-477.

MACIEL, A. C. \& MENDONÇA, M. A. R. Bibliotecas como Organizações. Rio de Janeiro: Interciência; Niterói: Intertexto, 2006.

MINTZBERG, H.; AHLSTRAND, B. \& LAMPEL, J. Safári de Estratégia: um Roteiro pela Selva do Planejamento Estratégico. Porto Alegre: Bookman, 2007.

RICHARTZ, T. "Metodologia Ativa: a Importância da Pesquisa na Formação de Professores". Revista da Universidade Vale do Rio Verde, Três Corações, vol. 13, n. 1, 2015, pp. 296-304.

RUIZ-PRIMO, M. A.; BRIGGS, D.; IVERSON, H.; TALBOT, R.; SHEPARD, L. A. "Impact of Undergraduate Science Course Innovations on Learning". Science, vol. 331, n. 6022, mar. 2011, pp. 1269-1270.

SODERDAHL, P. A. "Library Classroom Renovated as an Active Learning Classroom". Library Hi Tech, vol. 29, n. 1, 2011, pp. 83-90.

THIOLLENT, M. Metodologia da Pesquisa-Ação. 17. ed. São Paulo: Cortez, 2009.

VILLAR, E. G. \& WALTER, S. A. "O Conteúdo das Disciplinas deEstratégianos Programas dePós-Graduação Stricto Sensu em Administração no Brasil". Encontro da ANPAD, 38, 2014, Rio de Janeiro. Anais... Rio de Janeiro: ANPAD, 2014, pp. 1-16. Disponível em: \http://www. anpad.org.br/admin/pdf/2014_EnANPAD_ESO1159. pdÐ . Acessado em 18 mar. 2016. 